\title{
GRAVITATIONAL COLLAPSE AND FRAGMENTATION IN MOLECULAR CLOUDS WITH ADAPTIVE MESH REFINEMENT HYDRODYNAMICS
}

\author{
RICHARD I. KLEIN, ROBERT T. FISHER, CHRISTOPHER F. MCKEE AND \\ J. KELLY TRUELOVE \\ Astronomy Department, University of California \\ 601 Campbell Hall \\ Berkeley, Ca. 94720
}

\begin{abstract}
We describe a powerful methodology for numerical solution of 3 -D self-gravitational hydrodynamics problems with extremely high resolution. Our method utilizes the technique of local adaptive mesh refinement (AMR), employing multiple grids at multiple levels of resolution. These grids are automatically and dynamically added and removed as necessary to maintain adequate resolution. This technology allows for the solution of problems in a manner that is both more efficient and more versatile than other fixed and variable resolution methods. The application of AMR to simulate the collapse and fragmentation of a molecular cloud, a key step in star formation, is discussed. Such simulations involve many orders of magnitude of variation in length scale as fragments form. In this paper we briefly describe the methodology and present an illustrative application for nonisothermal cloud collapse. We describe the numerical Jeans condition, a criterion for stability of self-gravitational hydrodynamics problems. We show the first well-resolved nonisothermal evolutionary sequence beginning with a perturbed dense molecular cloud core that leads to the formation of a binary system consisting of protostellar cores surrounded by distinct protostellar disks. The scale of the disks, of order $100 \mathrm{AU}$, is consistent with observations of gaseous disks surrounding single T-Tauri stars and debris disks surrounding systems such as $\beta$ Pictoris.
\end{abstract}

RIK is also affiliated with Lawrence Livermore National Laboratories, L-023, Livermore, California, 94550. 


\section{INTRODUCTION}

Star formation ultimately involves the collapse of an interstellar molecular cloud core from an initial density of $\rho \leq 10^{-19} \mathrm{~g} \mathrm{~cm}^{-3}$ and size $\simeq 10^{17} \mathrm{~cm}$ to a final young stellar object of density $\rho \geq 10^{-1} \mathrm{~g} \mathrm{~cm}^{-3}$ and size $\simeq 10^{11} \mathrm{~cm}$. The collapse of a portion of a cloud may lead to fragmentation, which is crucial to establishing key parameters of binary stars: the distributions of mass ratios, periods, and orbital eccentricities. It may also be essential to the formation of larger groups of stars and to the determination of the stellar initial mass function. However, this enormous dynamic range presents a formidable obstacle to obtaining an accurate numerical solution, as the flow must remain well-resolved throughout the evolution. Fixed-resolution methods cannot be used to simulate such a 3 -D collapse in a practical amount of time using current computers.

In order to treat the enormous dynamic range presented by gravitational collapse calculations and address the problems associated with alternative methods such as smoothed particle hydrodynamics (SPH) and static nested-grid codes (Burkert \& Bodenehimer, 1993), we have developed a 3-D self-gravitational adaptive mesh refinement (AMR) code. Specifically, SPH suffers from two major drawbacks. First, it is typically very diffusive, and has great difficulty tracking shocks and contact discontinuities accurately in multi-dimensional flows. Second, although it is an adaptive method, it is only adaptive in the sense that the local smoothing length $h$ tracks mass in a Lagrangian sense, i.e. in $3-\mathrm{D}, h \propto \rho^{-1 / 3}$. Furthermore, in the context of isothermal collapse, in which the Jeans length $\lambda_{J}$ scales as $\lambda_{J} \propto \rho^{-1 / 2}$, the effective resolution, specified by the ratio $\lambda_{J} / h$ varies as $\rho^{-1 / 6}$ - the effective resolution of the calculation is degraded as collapse proceeds, and if the collapse is not arrested first, the calculation will eventually become underresolved. Given that in SPH one cannot choose a priori which regions of the flow need further refinement, one's only recourse to increasing the resolution of a calculation is to increase the total number of particles. Lastly,

static nested-grid codes suffer from the drawback of imposing a static set of grids from the beginning of the calculation, and hence are unable to resolve arbitrarily-located fragments.

Our AMR method dynamically resizes and repositions grids and inserts new, finer ones within them according to adjustable refinement criteria (unlike the static nested grid scheme of Burkert \& Bodenehimer 1993). We have applied our method to great effect; prior to the addition of selfgravity, we have used AMR to study astrophysical problems including the interaction of supernova blastwaves with interstellar clouds (Klein, McKee, \& Colella 1994; Klein, \& McKee 1994; Klein, McKee, \& Woods 1995), X-ray heated coronae and winds from accretion disks (Woods et al., 1996), and the 
collision of interstellar clouds (Klein \& Woods, 1998) with unprecedented high resolution.

We present the methodology behind our 3-D AMR self-gravity code and describe application of our work to the collapse and fragmentation of molecular clouds. Our discussion closely follows Truelove et al. 1998 and Klein 1998.

\section{METHODOLOGY}

\subsection{SELF-GRAVITATIONAL HYDRODYNAMICS}

The basic governing equations of our model are the Euler equations of hydrodynamics in 3-D, including effects of self-gravitation, and Poisson's equation for the gravitational potential. We term these the gravitational hydrodynamics (GHD) equations. The total non-gravitational energy per unit mass, $E$, is related to the internal energy per unit mass, $e$, by $E=e+\frac{1}{2} \mathbf{v}^{2}$. In turn, $P$ is defined by an equation of state. In our isothermal work, we adopt the ideal gas law $P=(\gamma-1) \rho e$. In recent work (Fisher, Klein, \& McKee 1998) we have used a nonisothermal EOS that combines isothermal and nonisothermal components such that $P(\rho)=c_{s}^{2} \rho+K \rho^{\gamma}$ where $\gamma=5 / 3$ and $K$ is chosen so that the isothermal and adiabatic components balance at a critical density determined by detailed 1-D radiation-hydrodynamic calculations of Masunaga, Miyama, \& Inutsuka (1998). A typical critical density for this transition is $5 \times 10^{-14} \mathrm{~g} \mathrm{~cm}^{-3}$. As written above, there are eight equations in eight unknowns. We assume periodic boundary conditions on these equations.

Our code methodology consists of three components to efficiently solve this system. The first component is a hyperbolic solver that employs an implementation of the Godunov method (see Colella \& Woodward 1984 and LeVeque 1992) for solution of the Euler equations of gas dynamics. The second major component of our code methodology is an elliptic solver that utilizes an AMR multigrid method to solve Poisson's equation. These elements operate within the third component, an adaptive mesh refinement framework.

\subsection{HYPERBOLIC SOLVER}

\subsubsection{Overview of Godunov Implementation}

To solve the full 3-D system of hydrodynamics equations, we use an operatorsplit method in which we solve in each coordinate direction independently in a cyclic sequence. In 3 -D, this operator splitting can be written schematically as

$$
\mathbf{Q}^{n+2}=L_{x} L_{y} L_{z}\left[L_{z} L_{y} L_{x}\left(\mathbf{Q}^{n}\right)\right]
$$


where the superscript $n$ indicates the $n^{\text {th }}$ time level, $\mathbf{Q}^{n}$ indicates the state vector at the $n^{\text {th }}$ time level, and $L_{i}$ is the update operator in the $i^{\text {th }}$ coordinate direction based on the higher-order Godunov method described by Colella \& Woodward (1984) and Bell, Colella, \& Trangenstein (1989). That is, each three-dimensional update step from $t^{n}$ to $t^{n+1}$ consists of 3 onedimensional update steps, one per coordinate direction. At the end of the cycle from $t^{n}$ to $t^{n+2}$, the solution is second-order accurate. The interested reader can refer to Truelove et al. (1998) and Klein (1998) for the details of the scheme.

\subsubsection{AMR Considerations and AMR Multigrid}

When solving a hyperbolic system on a hierarchy of computational grids, care must be taken that the solutions on finer grids are reflected on the neighboring and underlying coarser grids. Our code uses refluxing and averaging down procedures described in detail by Berger \& Colella (1989) to update the solutions on these coarser grids to account for solutions on the finer grids. In refluxing, the solution in a coarse cell adjacent to a fine grid is updated after the fine grids have been advanced in time by adding the effects of a differential flux acting over $\Delta t$ at its face adjacent to the refined region. This differential flux is equal to the difference between the flux at this face as computed on the coarse grid and the sum of the fluxes at this face as computed on the fine grid. The solution in a refined coarse cell is simply overwritten by averaging down the solutions on the fine cells it contains.

In contrast to the hyperbolic solution, in which only neighboring grids communicate at each time step, the solution of Poisson's equation for the gravitational potential is a much more tightly coupled process. The multigrid method is a natural choice to use as an element of the elliptic solver in an AMR calculation (Truelove et al. 1998).

\subsection{AMR FRAMEWORK AND THE REFINEMENT CRITERION}

As discussed above, the basic hydrodynamic method we use is a higherorder extension of Godunov's method of a type discussed in Colella \& Woodward (1984). This algorithm is second-order accurate for smooth flow problems, and has a robust and accurate treatment of discontinuities (Klein, McKee, \& Colella, 1994). We further enhance the efficiency and accuracy of our calculations by using local adaptive mesh refinement.

The version of the algorithm used for the present work is rather elaborate; a detailed description is given in Berger \& Colella 1989.

AMR contains five separate components. The error estimator is used to estimate local truncation error and will be described subsequently. The 
grid generator creates fine grid patches that cover the regions that need refinement. Data structure routines manage the grid hierarchy allowing access to the individual patches. Interpolation routines initialize a solution on a newly created fine grid and also provide the boundary conditions for integrating the fine grids. Flux correction routines ensure conservation at grid interfaces by modifying the coarse grid solution for coarse cells that are adjacent to a fine grid.

In AMR, the computational volume consists of a hierarchical grid structure. A base Level 0 grid fills the computational volume, discretizing it on a rectangular grid with a resolution of $\Delta x_{0}$ in each direction. Multiple Level 1 grids of finer resolution $\Delta x_{1}=\Delta x_{0} / r_{1}$ may be embedded within it, where $r_{1}=4$ is a typical choice. Higher levels may be defined embedded in underlying levels in a similar fashion. Grids at Level $L$ always span an integral number of cells at Level $L-1$, i.e., partial cell refinement is not permitted. Furthermore, an interior grid at Level $L$ is always nested within a grid at Level $L-1$ such that there is a buffer region of Level $L-1$ cells surrounding it. In other words, a grid at Level $L$ within a grid at Level $L-1$ never shares a boundary with the Level $L-1$ grid, unless it shares a boundary with the computational domain.

An integration step on this adaptive grid proceeds as follows. We are using a single timestep for all grids, determined from the most stringent timestep over the entire physical domain. The updating of the data on the locally refined grid structure is organized around the grouping of cells into rectangular grid patches, each typically containing hundred to several thousand grid cells. The AMR code passes a grid patch to an integration routine which returns the updated cell values as well as the edge-centered conservative fluxes used in the update. The overheads in both CPU and memory associated with the adaptive mesh structure have been kept quite small, relative to irregular grid schemes. Typically, $80 \%-90 \%$ of the total execution time is spent advancing cells in time using the hydrodynamic and elliptic solvers, while the memory required is that needed to store two copies of the solution on all of the grids. These overheads are low because they are determined by the number of rectangles into which the AMR solution has been divided; as opposed to being determined by the number of grid cells, as is the case with irregular grid adaptive algorithms.

A key component of an AMR code is the grid generator. In our code, this procedure is broken into two steps. In the first step, a specified property is measured in each cell, and cells requiring refinement are flagged. In the second step, the distribution of flagged cells is analyzed to determine the number, sizes, and locations of grids to be inserted at the next finer level of resolution. These finer grids will always include every cell that was flagged for refinement, but they may also include additional cells that were not 
flagged. The degree to which the refinement is concentrated in the cells that require it is termed the grid efficiency. The grid efficiency is minimal when the smallest rectangular solid containing all flagged cells is refined. The grid efficiency is maximal when the only cells refined are those that were flagged.

Our general process for deciding upon a suitable level of refinement depends on a method for estimating the local truncation error of the Godunov scheme; this error estimator determines where the solution accuracy is insufficient. We estimate truncation error using Richardson extrapolation; we take data on the grid where the error is being estimated and evolve two timesteps. This solution is then combined with the data on a grid spatially coarsened by a factor of two and integrated for one timestep. The local truncation error can be shown to be proportional to the normed functional that is the difference of these two operations.

In the nonisothermal calculations presented in this paper and in the isothermal calculations of Truelove et al. (1997), refinement to Level 2 and above is controlled by application of the Jeans condition. This criterion can be augmented or replaced by others as necessary. The Jeans condition used to make the cell-by-cell refinement decision is a physically motivated resolution constraint discussed by Truelove et al. (1997). Jeans (Jeans 1902 and Jeans 1928) analyzed the linearized equations of 1-D isothermal GHD for a medium of infinite extent and found that perturbations on scales larger than the Jeans length, $\lambda_{J} \equiv\left(\frac{\pi c_{s}^{2}}{G \rho}\right)^{1 / 2}$, are unstable. Thermal pressure cannot resist the self-gravity of a perturbation larger than $\lambda_{J}$, and runaway collapse results. Truelove et al. (1997) showed that the errors generated by numerical GHD solvers can act as unstable perturbations to the flow. In a simulation with variable resolution, cell-scale errors introduced in regions of coarser resolution can be advected to regions of finer resolution, affording these errors the opportunity to grow. The unstable collapse of numerical perturbations can lead to substantial fragments, a process termed artificial fragmentation, which can be avoided if $\lambda_{J}$ is sufficiently resolved. Defining the Jeans number resolution of $\lambda_{J}$. Defining the Jeans number $J \equiv \frac{\Delta x}{\lambda_{J}}$, Truelove et al. (1997) found keeping $J \leq 0.25$ avoided artificial fragmentation in isothermal evolution of a collapse spanning 7 decades of density, the approximate range separating typical molecular cloud cores from nonisothermal protostellar fragments. The constraint that $\lambda_{J}$ be resolved is termed the Jeans condition. Although it has been shown to be necessary only for isothermal evolution, it is reasonable to expect that it is necessary (although not necessarily sufficient) for nonisothermal collapse as well.

As a side effect of confining cell-sized perturbations to a length scale at which they are thermally damped, resolution of $\lambda_{J}$ also ensures that gradi- 
ents developed in isothermal flow by gravity are well resolved. Formation of structure on scales of $\lambda_{J}$ and larger is a general feature of self-gravitational isothermal GHD flow since smaller fluctuations are damped but larger ones collapse. For example, in the self-similar solution for isothermal cylindrical collapse (Inutsuka \& Miyama, 1992), the radial scale height of the cylinder is $\lambda_{J}$. Lack of resolution of gradients within simulated flow triggers the injection of artificial viscosity, which is generally intended to be introduced in small amounts only for numerical stability (in our Godunov scheme) or shock mediation (in many common non-Godunov schemes). Introducing excess amounts of artificial viscosity renders the problem solved different from the inviscid problem posed. Continuous resolution of $\lambda_{J}$, however, keeps the flow inviscid and prevents artificial slowing of gravitational collapse. It is important to note, however, that the Jeans condition is a necessary but not, in general, sufficient condition to ensure convergence.

The Jeans condition on $\Delta x$ fundamentally differs from the Courant condition on $\Delta t$, although at first the two conditions might appear analogous. The Courant condition arises from a modal stability analysis of finite difference equations derived from the Euler partial differential equations (PDEs) of hydrodynamics (see, e.g., Richtmyer \& Morton 1967). It is entirely a consequence of the finite differencing and has no physical counterpart in the PDEs. A modal stability analysis of finite difference equations derived from the GHD PDEs does not yield the Jeans condition, but rather a generalized Courant condition that includes effects of gravity (Truelove et al. 1998).

\section{APPLICATIONS TO ROTATING MOLECULAR CLOUDS}

In this section we briefly describe recent high-resolution results we have obtained with AMR for the gravitational collapse of nonisothermal, rotating, uniformly dense clouds (Fisher, Klein, \& McKee 1998). Isothermal centrally condensed clouds (Truelove et al., 1997) and isothermal uniform clouds (Truelove et al., 1998) have been previously discussed and we refer the reader to these papers.

The uniform cloud is an idealized model of an astrophysical cloud but is very useful as a first approximation. The initial isothermal configuration of the uniform cloud may be completely parameterized by the dimensionless energy ratios $\alpha \equiv E_{\text {thermal }} /\left|E_{\text {gravitational }}\right|$ and $\beta_{\Omega} \equiv E_{\text {rotational }} /\left|E_{\text {gravitational }}\right|$.

Burkert \& Bodenehimer 1993 studied a uniform cloud, with $M=1 M_{\odot}$ and $R=5 \times 10^{16} \mathrm{~cm}$, which give $\rho_{0}=10^{-17.4} \mathrm{~g} \mathrm{~cm}^{-3}$. Its energy ratios are $\alpha=0.26$ and $\beta_{\Omega}=0.16$. The isothermal uniform cloud thus has $c_{s}=$ $0.167 \mathrm{~km} \mathrm{~s}^{-1}$ and a rotation rate $\Omega=7.2 \times 10^{-13} \mathrm{rad} \mathrm{s}^{-1}$. The cloud is perturbed by $\rho \rightarrow \rho \times[1+0.1 \cos (2 \phi)]$, a $10 \% m=2$ mode seed perturbation. 
This cloud begins with $\lambda_{J}=1.17 R$. We have used an initial resolution of $R_{32}$ and dynamically refined so as to ensure $J_{\max }=0.25$. In the initial configuration, the fiducial radius beyond which cloud gravity dominates perturbation pressure is $r_{\text {crit }}=0.14 R$, so that with $R_{32}$ we still linearly resolve this scale with more than 4 cells.

\subsection{NON-ISOTHERMAL UNIFORM CLOUDS}

In recent work using high resolution AMR (Fisher, Klein, \& McKee 1998), we followed the collapse of an initially rigidly rotating, uniform isothermal cloud. We used a two-component barotropic equation of state which makes the transition from isothermal to polytropic in a smooth fashion. The initial conditions are identical to the Burkert \& Bodenehimer 1993 isothermal uniform cloud perturbed by a $10 \% m=2$ mode. In the following we discuss the evolution of this nonisothermal collapse. While a similar calculation has been recently studied by Bate\&Burkert (1997), to our knowledge, none of the calculations in the literature to date have been able to follow the subsequent evolution of the fragments over dynamical (orbital) timescales, while still adhering to the Jeans criterion. However, to be fully certain of the reality of the fragmentation, it is still necessary to do a convergence study by decreasing the Jeans number (Truelove et al. 1997), which we have not yet done for this calculation.

Figure 1 represents a 2-D slice of log density through the equatorial plane $t=1.41 \times 10^{12} \mathrm{~s}$ after start of the collapse. The cloud has initially collapsed to an isothermal disk, and an elongated filamentary bar with the first signs of fragmentation apparent. A strong isothermal shock above the plane of the disk is soon established. After $t=1.46 \times 10^{12} \mathrm{~s}$ (Figure 2) the isothermal bar becomes optically thick, and the accretion flow onto the bar is arrested, resulting in the growth of non-axisymmetric perturbations in the bar. Fragmentation in the bar results in the formation of binary spherical cores connected by a prominent bar. The core-bar system is embeddded in a two-armed spiral, derivative of the original $m=2$ perturbation. The binary separation decreases as the cores increase their mass by direct accretion from the low-angular momentum material of the bar, eventually leading to the dissipation of the bar. Figure 3 shows a snapshot of log density about 0.5 rotation periods later at $t=1.51 \times 10^{12} \mathrm{~s}$. Protostellar disks have now formed around the cores with the cores at their closest orbital separation $\sim 44 \mathrm{AU}$. The disks accrete gas directly from the long spiral arms. Each core has $0.08 M_{\odot}$ with a radius $\sim 10 \mathrm{AU}$. The masses and radii of these first collapse cores are in good agreement with the detailed 1-D radiationhydrodynamic calculations of Masunaga, Miyama, \& Inutsuka (1998). The

core accretion luminosity $\sim 2 \times 10^{31} \mathrm{ergs}^{-1}$ obtained in the 3 -D collapse 
calculations is considerably less than that found in the 1-D simulations, $\sim 4 \times 10^{32} \mathrm{ergs}^{-1}$, due to the large angular momentum barrier in the $3-\mathrm{D}$ simulations, resulting in slower accretion onto the protostellar cores.

It is important to point out that the initial rapid growth of the cores is due to accretion of matter from the bar. As the bar dissipates, the cores have ended their first phase of growth and proceed to grow more slowly by direct accretion from the surrounding protostellar disks. At $t=1.52 \times 10^{12}$ $\mathrm{s}$ (Figure 4) the cores begin to separate and the surrounding disks become morphologically distinct. The protostellar disks and cores become a fully detached binary by $t=1.56 \times 10^{12} \mathrm{~s}$ (Figure 5 ) with the disks attached to the long spiral. The scale of the disks, of order $100 \mathrm{AU}$, is consistent with observations of gaseous disks surrounding single T-Tauri stars (Sargent \& Beckwith 1994) and debris disks surrounding systems such as $\beta$ Pictoris (Artymowicz 1997). When we stopped our calculations, at $t=1.6 \times 10^{12}$ $\mathrm{s}$ (Figure 6 ) the binary protostellar disks/core system has moved back into the surrounding spiral arms. The cores have $20 \%$ of the mass of the initial cloud and the arms have $27 \%$ of mass of the initial cloud at this time. The protostellar disks comprise about $2 \%$ of the cloud mass.

\section{CONCLUSIONS}

In this paper we have described a powerful new method for numerical solution of 3-D self-gravitational hydrodynamic problems. This method combines a Godunov hydrodynamics integrator with a multigrid gravity solver in an adaptive mesh refinement framework. Guided by the Jeans condition for isothermal problems, and the use of Richardson extrapolation, AMR efficiently expends computational resources only when and where the features of the flow demand it. We presented results of this methodology applied to the collapse and fragmentation of nonisothermal molecular clouds that are both initially uniform and centrally condensed, evolved over 8-9 decades of collapse and subject to initial $m=2$ mode perturbations. We found binary fragmentation results in good agreement with published calculations after evolution over the first half of this logarithmic range. By automatically inserting ever finer grids to maintain resolution of the Jeans length, these calculations did not generate the substantial artificial viscosity found in published calculations that used fixed finest resolutions. We have shown the first well-resolved formation sequence of protostellar disks surrounding a pair of binary cores, and discussed the role of the bar connecting the binary pair in the accretion onto the cores. The disks formed are consistent with observations of gaseous disks surrounding single T-Tauri stars and debris disks surrounding systems such as $\beta$ Pictoris. 


\section{Acknowledgements}

Research on star formation for RIK and CFM is supported by a grant from NASA's Astrophysics Theory Program to the Center for Star Formation Studies. RIK acknowledges additional support under the auspices of the US Department of Energy at the Lawrence Livermore National Laboratory under contract W-7405-Eng-48. CFM's research is supported in part by NSF grant AST9530480. We wish to thank the Pittsburgh Supercomputing Center for provision of Cray C90 resources through grant AST940011P.

\section{References}

Artymowicz, P. 1997, Ann. Rev. Planet. Sci., 25, 175

Bate, M.R.\& Burkert, A. 1997, M.N.R.A.S., 288, 1060

Bell, J.B., Colella, P., \& Trangenstein, J. 1989, Journal of Computational Physics, 82, 362

Berger, M.J. \& Colella, P. 1989, Journal of Computational Physics, 82, 64

Berger, M.J. \& Oliger, J. 1984, Journal of Computational Physics, 53, 484

Boss, A.P. 1991, Nature, 351, 298

Boss, A.P. 1996, ApJ, 468, 231

Burkert, A. \& Bodenheimer, P. 1993, M.N.R.A.S., 264, 798

Colella, P. \& Glaz, H.M. 1985, Journal of Computational Physics, 59, 264

Colella, P. \& Woodward, P.R. 1984, Journal of Computational Physics, 54, 174

Fisher, R., Klein, R., \& McKee, C. 1998, in preparation

Inutsuka, S. \& Miyama, S.M. 1992, ApJ, 388, 392

Inutsuka, S. \& Miyama, S.M. 1997, ApJ, 480, 681

Jeans, J.H. 1902, Phil. Trans. A, 199, 1

Jeans, J.H. 1928, Astronomy \& Cosmogony, (London: Cambridge University Press) 1993, A\&A, 273, 175

Klein, R.I. \& McKee, C.F. 1994, in Numerical Simulations in Astrophysics, ed. J. Franco et al. (New York: Cambridge), 251

Klein, R.I., McKee, C.F., \& Colella, P. 1994, ApJ, 420, 213

Klein, R.I., McKee, C.F., \& Woods, D.T. 1995, in Physics of the Interstellar Medium and Intergalactic Medium, ed. A. Ferrara et al. (San Francisco: Astronomical Society of the Pacific), 366

Klein, R.I. \& Woods, D.T. 1998, ApJ, 497,777

Klein, R.I. 1998, Journal of Computational and Applied Mathematics, ed. H. Riffert (Elsevier Press), in press

LeVeque, R.J. 1992, Numerical Methods for Conservation Laws, (Boston: Birkhauser Verlag)

Masunaga, H., Miyama, S. \& Inutsuka, S. 1998, ApJ, 495, 346

Richtmyer, R.D. \& Morton, K.W. 1967, Difference Methods for Initial-Value Problems, (New York: Interscience)

Sargent, A.I, \& Beckwith, S.V.W., 1994, Astroph. and Space Sci., 212, 181.

Truelove, J.K., Klein, R.I., McKee, C.F., Holliman, J.H., II, Howell, L.H., \& Greenough, J.A. 1997, ApJ, 489L,179

Truelove, J.K., Klein, R.I., McKee, C.F., Holliman, J.H., II, Howell, L.H., Greenough, J.A., \& Woods, D.T. 1998, ApJ,495,821

Winkler, K.-H.A. \& Newmanm, M.J. 1980, ApJ, 238, 311

Woods, D.T., Klein, R.I., Castor, J.I., McKee, C.F., \& Bell, J. 1996, ApJ, 461, 767 
Uniform cloud with $10 \% \mathrm{~m}=2$ perturbation.

$X Y$ slice of $\log$ density at equatorial plane

Resolution level 3 of 3

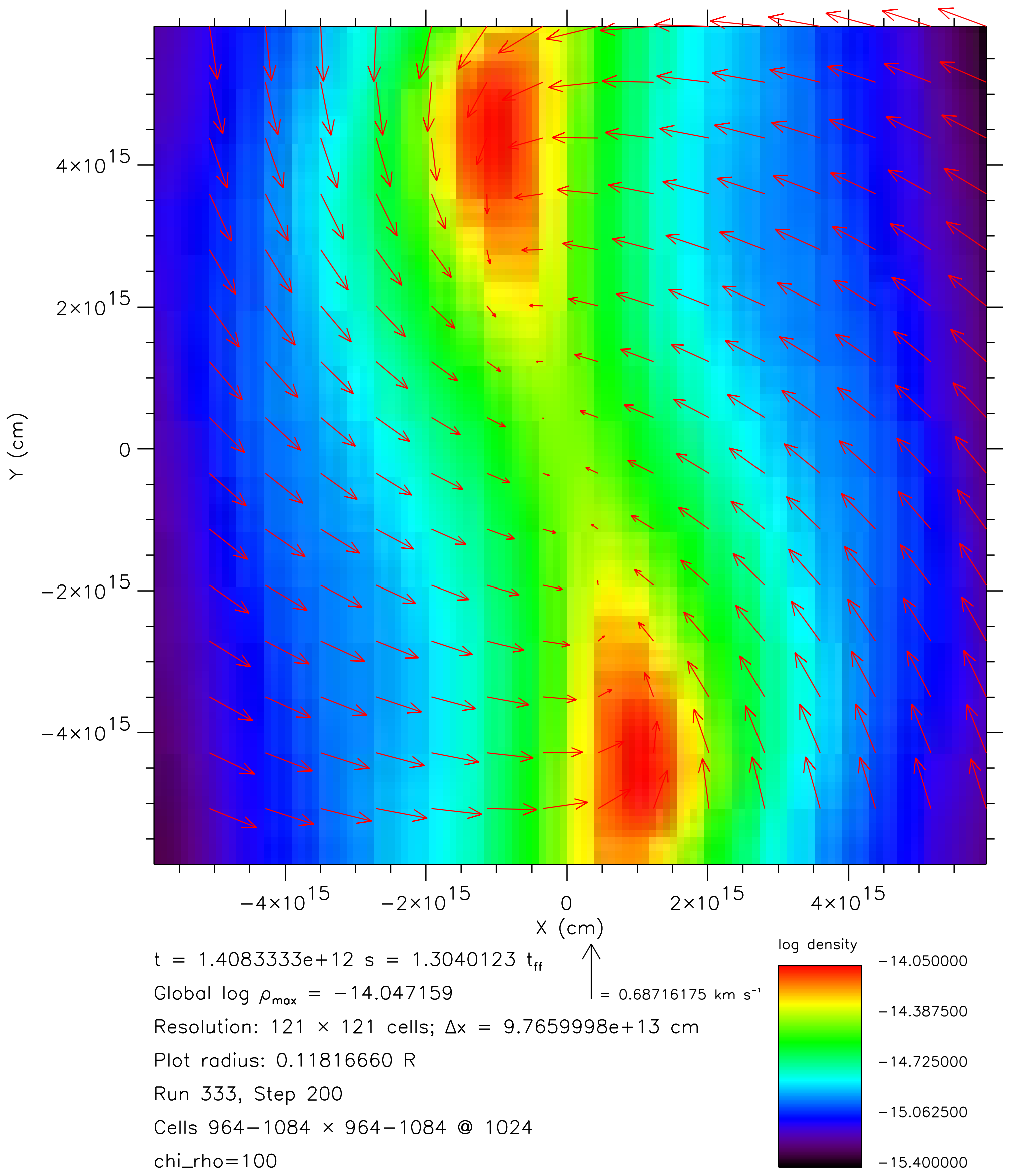


Uniform cloud with $10 \% \mathrm{~m}=2$ perturbation.

$X Y$ slice of log density at equatorial plane

Resolution level 4 of 4

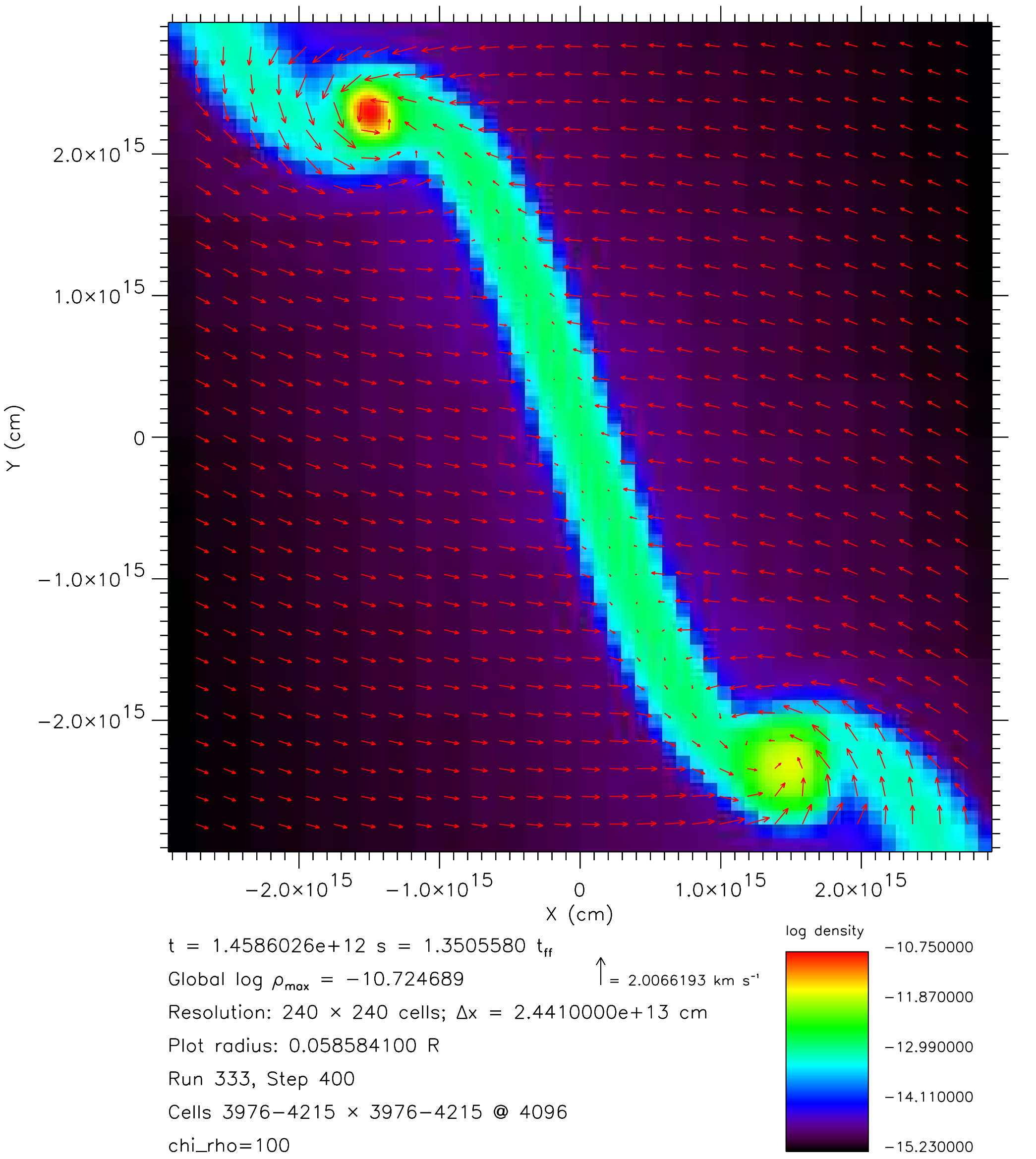


Uniform cloud with $10 \% \mathrm{~m}=2$ perturbation.

$X Y$ slice of $\log$ density at equatorial plane

Resolution level 4 of 4

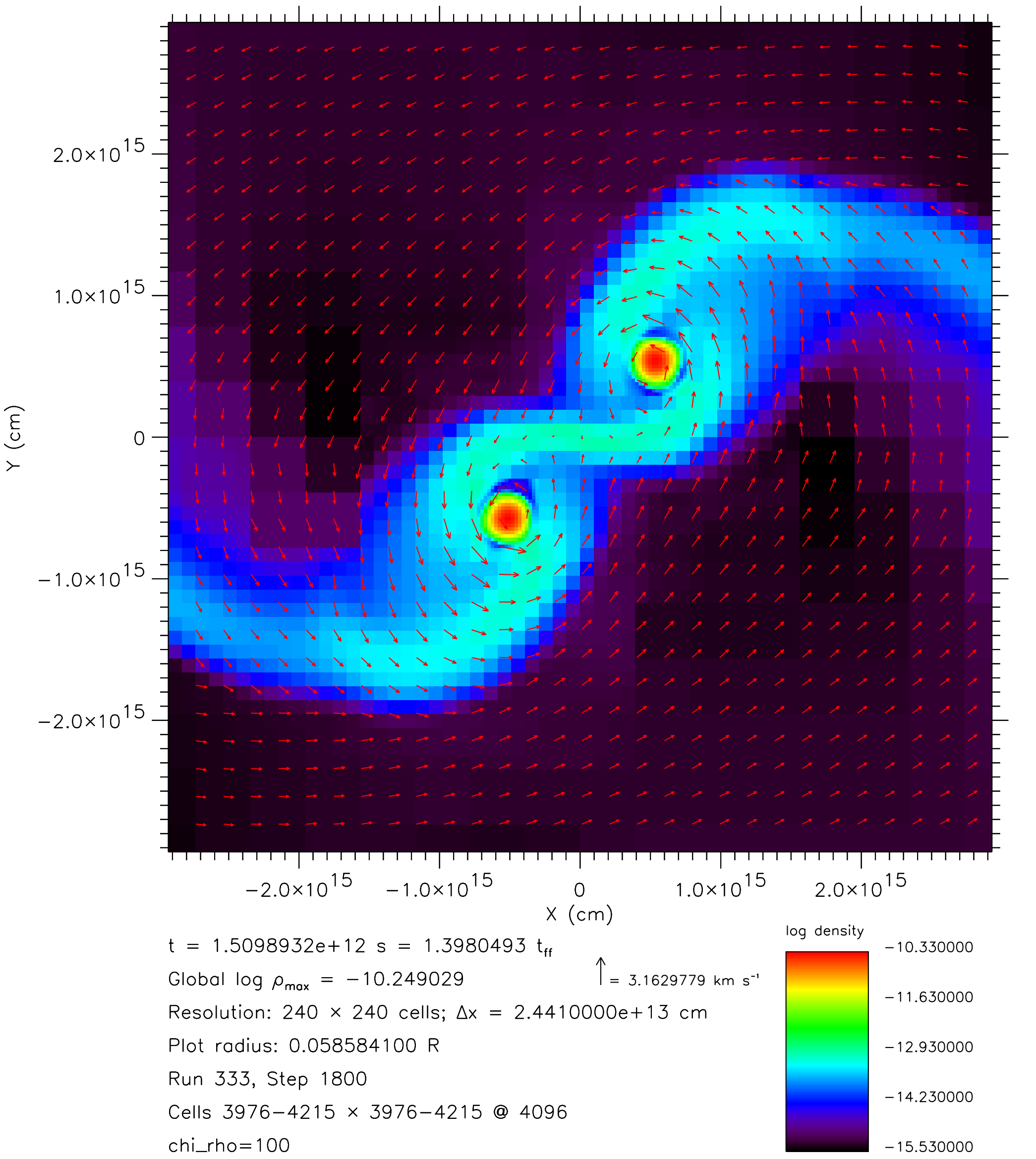


Uniform cloud with $10 \% \mathrm{~m}=2$ perturbation.

$X Y$ slice of $\log$ density at equatorial plane

Resolution level 4 of 4

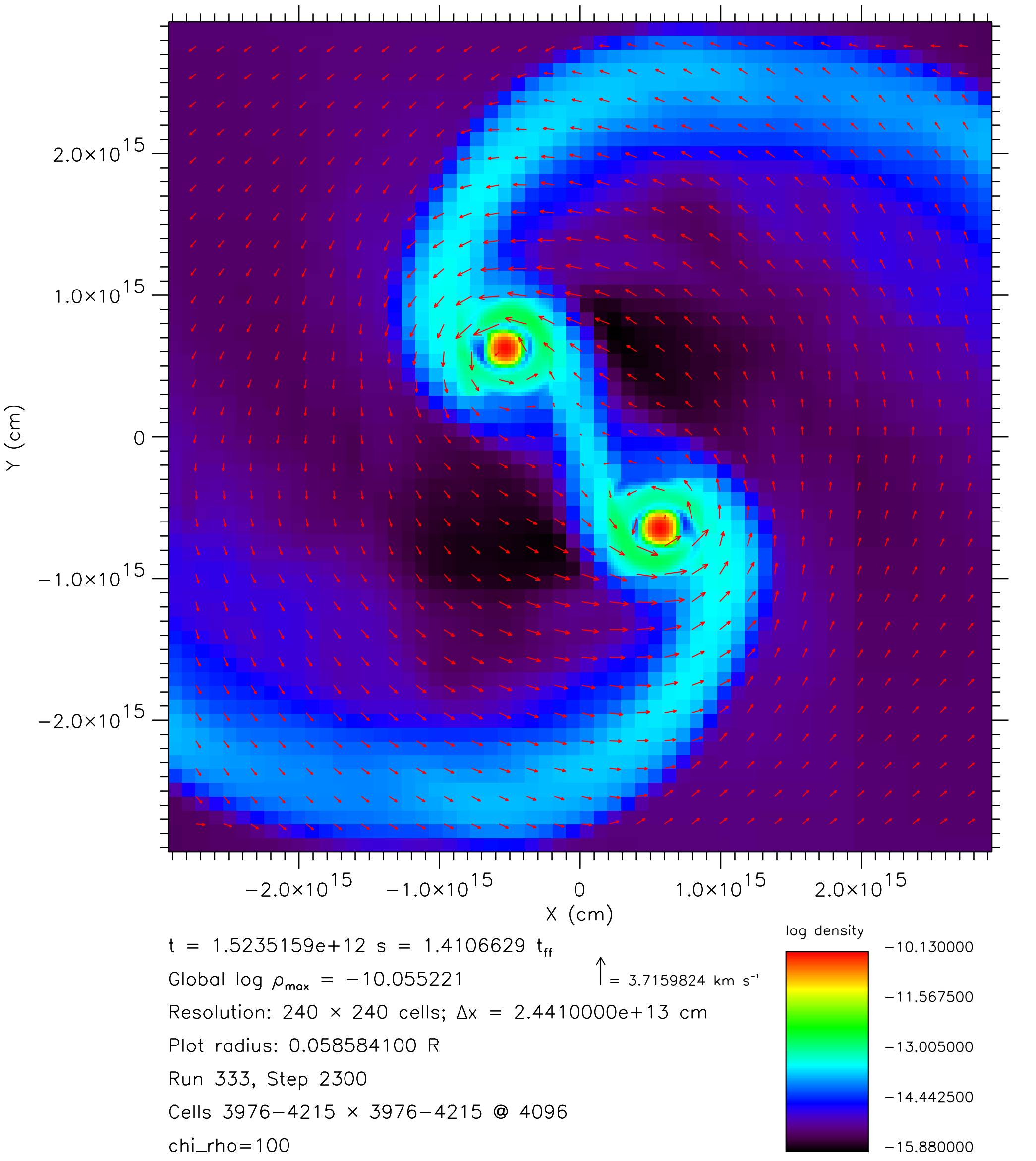


Uniform cloud with $10 \% \mathrm{~m}=2$ perturbation.

$X Y$ slice of $\log$ density at equatorial plane

Resolution level 4 of 4

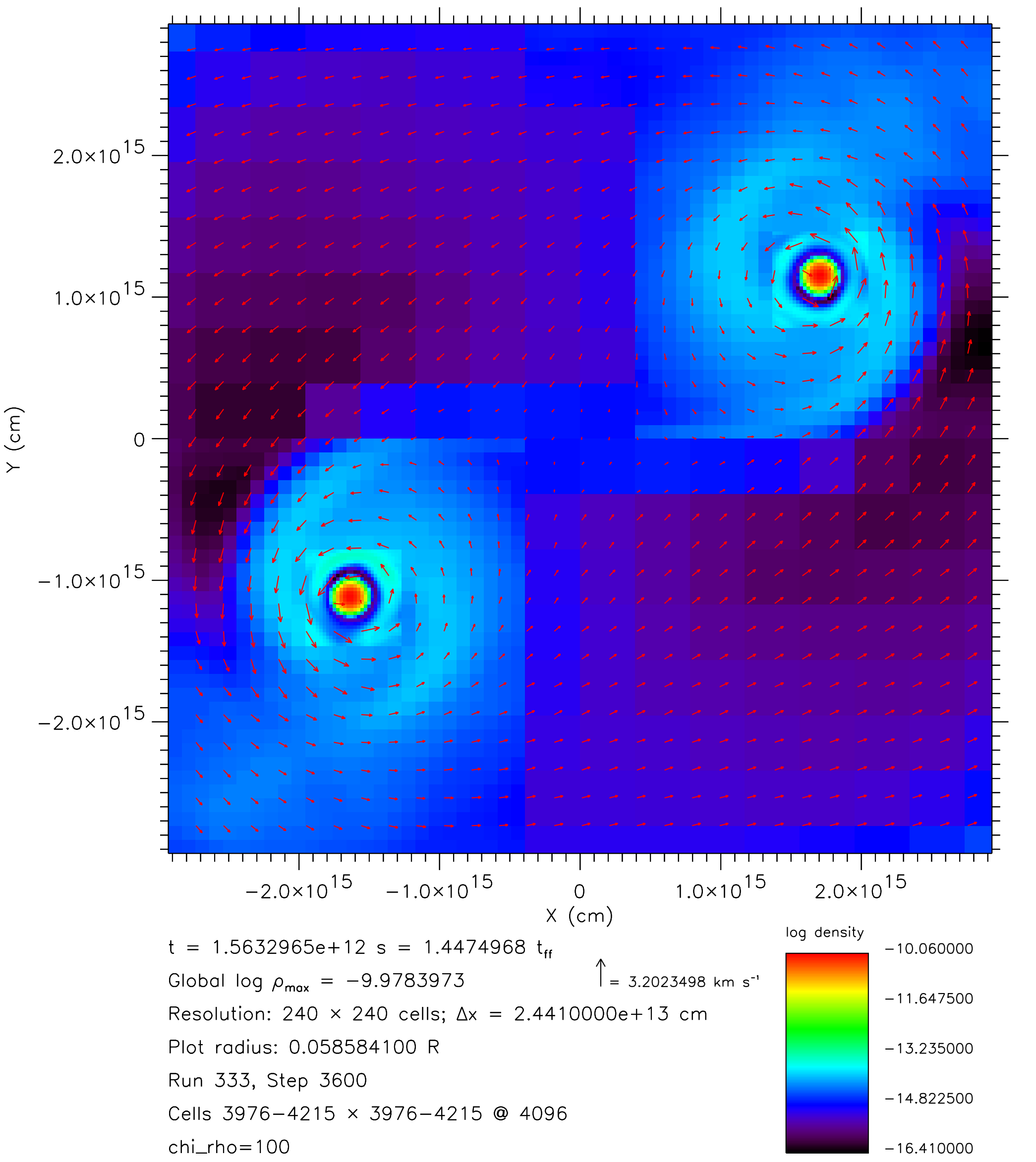


Uniform cloud with $10 \% \mathrm{~m}=2$ perturbation.

$X Y$ slice of log density at equatorial plane

Resolution level 3 of 4

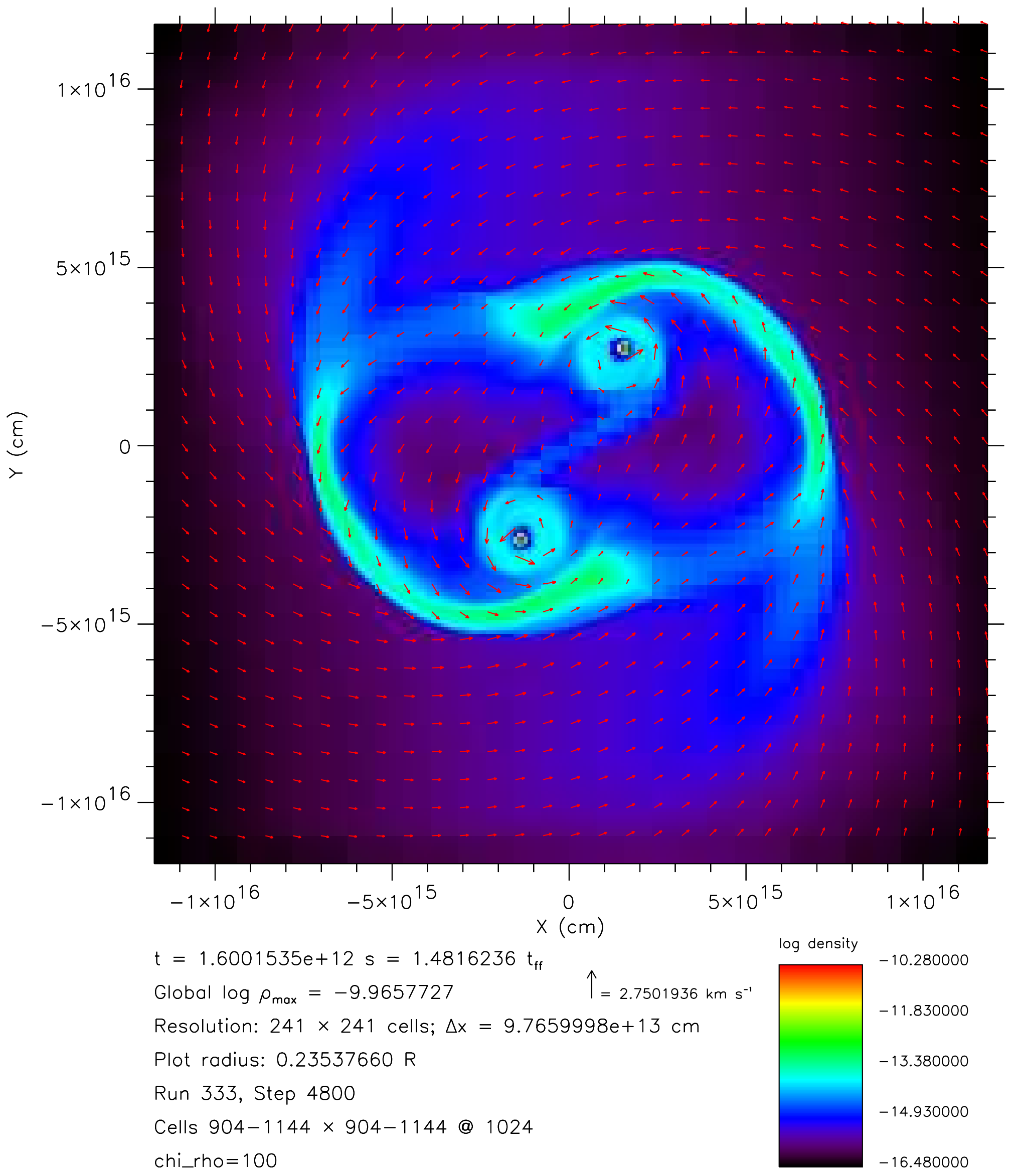

